\title{
Relationships Between C-Kit Expression and Mean Platelet Volume in Benign, Preneoplastic and Neoplastic Endometrium
}

\author{
Ibrahim Sehitoglu' ${ }^{1 *}$, Recep Bedir ${ }^{1}$, Ulku Mete Ural ${ }^{2}$, Hasan Gucer ${ }^{1}$, Cuneyt \\ Yurdakul $^{3}$, Medine Cumhur Cure ${ }^{4}$, Erkan Cure ${ }^{5}$, Suleyman Yuce ${ }^{6}$, Figen Kir \\ Sahin $^{2}$
}

\begin{abstract}
Background: $\mathrm{c}-\mathrm{Kit}$ is a proto-oncogene that encodes a tyrosine kinase receptor (CD117). Mean platelet volume (MPV) is a useful marker for demonstrating thrombocyte function. We aimed to investigate whether c-kit is expressed in benign, preneoplastic and neoplastic endometrial tissues and whether MPV has a relation with c-kit expression and its intensity. Materials and Methods: c-Kit expression was investigated immunohistochemically in 10 samples of normal endometrium $(n=10)$, simple endometrial hyperplasia $(5$ cases with atypia and 10 cases without atypia), complex endometrial hyperplasia (10 cases with atypia and 10 cases without atypia) and endometrial cancer (EC) (10 cases grade I and 10 cases grade II) and MPV of all cases was checked. Results: c-Kit expression was observed at very low rates in cases with normal endometrial tissues (NE) and in hyperplasia without atypia. c-Kit expression and immunostaining were strong in endometrial atypia and EC. MPV levels of complex atypical endometrial hyperplasia (CAEH) (p:0.002), EC grade I (ECG I) $($ p $<0.001)$ and EC grade II (ECG II) (p<0.001) were significantly elevated when compared with the NE group. Both c-kit expression and intensity of immunostaining had a positive correlation with MPV level. Conclusions: While c-kit expression and intensity of immunostaining were mildly positive in $\mathrm{NE}$ and hyperplasia without atypia, they were clearly observed in EC and hyperplasia with atypia. As c-kit expression is related to the mutagenesis a long-term followup may be needed in these cases. A high MPV level may be a good test for demonstrating c-kit expression and intensity of immunostaining.
\end{abstract}

Keywords: c-Kit - CD117-MPV - endometrial cancer - endometrial hyperplasia

Asian Pac J Cancer Prev, 16 (4), 1495-1499

\section{Introduction}

Endometrial cancer (EC) is one of the most common gynecological cancers in western world. The most common type of endometrial cancer is adenocarcinoma. The vast majority of the affected women are in perimenopausal or postmenopausal stages (Elmore et al., 2001). EC arises from endometrial hyperplasia that is related with obesity, inactivity and long estrogen exposure (Linkov et al., 2008). Some factors are thought to be responsible for the etiology of EC such as proinflammatory cytokines and tyrosine kinase family member as platelet derived growth factor (PDGF) (Dossus et al., 2013; Wang et al., 2014).

c-Kit - a member of PDGF family with a weight of $145-\mathrm{kd}$ - is a proto-oncogene that encodes tyrosine kinase receptor (CD117) and has a stem cell factor (SCF) as a ligand. In human it is localized on the q11-q12 region of $4^{\text {th }}$ chromosome (Yarden et al., 1987; d'Aurio et al.,
1988). It has been reported that high levels of c-kit and its ligand SCF may contribute to tumor development (Yilmaz et al., 2012). Thus c-kit expression has been investigated in various solid tumors and gynecological malignancy (Afsar et al., 2014; Xiao et al., 2014; Zhu et al., 2014). Additionally, it has been investigated in non-neoplastic, preneoplastic and neoplastic endometrial tissues. However, the results were reported to be different from each other (e.g in EC it was found to be 0\%-100\%) (Inoue et al., 1994; Scobie et al., 2003; Yilmaz et al., 2012)

Mean platelet volume (MPV) is a useful, simple and inexpensive marker that shows thrombocytes functions. In addition, MPV provides information about thrombocytes size (Cure et al., 2014). An investigation has been whether MPV level has a relation with various cancer types and it has been reported that MPV may be a useful marker for advanced stage EC (Oge et al., 2013). High MPV level indicates the presence of large and active thrombocytes in

${ }^{1}$ Department of Pathology, ${ }^{2}$ Department of Obstetrics and Gynecology, ${ }^{4}$ Department of Biochemistry, ${ }^{5}$ Department of Internal Medicine, Faculty of Medicine, Recep Tayyip Erdogan University, ${ }^{3}$ Department of Pathology, Rize Training and Research Hospital, Rize, ${ }^{6}$ Internal Medicine, Kumru State Hospital, Ordu, Turkey*For correspondence: sehitogluibrahim@gmail.com 
periphery. These thrombocytes express intensive PDGF, thromboxane A2, glycoprotein Ib and IIb/IIIa receptors (Park et al., 2002). These substances increase the risk of thrombosis in cancer patients, especially excessive release of PDGF shows a mutagenic effect (Wang et al., 2014). Both c-kit and PDGF are members of tyrosine kinase family. So excess c-kit and high MPV level may be effective in EC and tumor growth (Yilmaz et al., 2012; Oge et al., 2013). Additionally, estrogen and excessive cytokine production increase young and active thrombocyte release into periphery. The inhibition of c-kit and PDGF by tyrosine kinase inhibitors has been reported to be effective in the treatment of breast cancer (Weigel et al., 2012). We aimed to investigate whether c-kit is expressed in benign, preneoplastic and malign endometrial tissues and whether MPV has a relation with c-kit expression and its intensity.

\section{Materials and Methods}

\section{Patient selection and MPV measurement}

In this study, we included 65 patients who were diagnosed histopathologically after applying endometrial biopsy. The patients were subdivided into 7 groups according to the histopathological diagnosis of endometrial biopsy and the grading system of International Federation of Gynecology and Obstetrics (FIGO) scale. Group 1 normal endometrium (NE) $(n=10)$; group 2 simple atypical endometrial hyperplasia $(\mathrm{SAEH})(\mathrm{n}=5)$; group 3 simple endometrial hyperplasia without atypia (SEHA) $(n=10)$, group 4 complex atypical endometrial hyperplasia $(\mathrm{CAEH})(\mathrm{n}=10)$, group 5 complex endometrial hyperplasia without atypia (CEHA) $(n=10)$, group 6 endometrial cancer grade I (ECG I) $(n=10)$ and group 6 endometrial cancer grade II (ECG2) $(n=10)$. MPV measurement was performed using the Cell-Dyn Ruby analyzer (Abbott Diagnostics).

\section{Histopathological method}

Paraffin blocks of the patients were cut into 3 micrometer thickness on positively charged slide for immunohistochemical investigation. The sections were investigated immunohistochemically using CD117 (ready-to-use mouse monoclonal antibody, Biogenex, Fremont, CA, USA) primary antibody. The biotin-free, HRP multimer-based, hydrogen peroxide substrate and 3,3'-diaminobenzidine tetrahydrochloride (DAB) chromogen containing ultra-View ${ }^{\mathrm{TM}}$ Universal DAB
Detection Kit (Catalog number 760-091, Ventana Medical Systems, Tucson, AZ) and a full automated immunohistochemistry staining device (Ventana Bench Mark XT, Ventana Medical Systems, Tucson, AZ, USA) were applied for the IHC staining system. Immunohistochemical stain includes deparaffinization and antigen revealing procedures that were performed using Bench Mark XT fully automatic immunohistochemical staining device. Primer antibody CD117 was manually dribbled and incubated 30 minutes at $37^{\circ} \mathrm{C}$. Mayer's hematoxylin was used as a negative stain then the sections were investigated blindly by 2 pathologists using Olympus BX51 light microscopy. The intensity of the immunohistochemical staining was between +1 and +3 , the extent of staining was accepted as focal (10\% of cells), intermediate ( $>10$ and $<50 \%$ of cells), and diffuse $(>50 \%$ of cells) (Yilmaz et al., 2012)

\section{Statistical analysis}

All the results were given as mean \pm SD. Statistical analysis was performed using SPSS packet program (version 18.0, USA). The evaluation of immunostaining distribution and intensity of two by two groups was performed by Mann Whitney U test using. The hematologic evaluation was made by One Way Anova test and posthoc test using bonferroni. Correlation analysis was performed using Pearson correlation test. P level $<0.05$ was considered significant.

\section{Results}

\section{Immunostaining distribution}

While the majority of NE patients sections (70\%) had negative CD117 staining, only 3 cases (30\%) were observed to be focally stained (Figure 1A). When a comparison was made among the groups with simple hyperplasia while 4 cases of SEHA were not stained at all 5 cases were focally stained (Figure 1B). While there was no stain in 2 cases of SAEH only 3 cases of the other patients each of them was once stained diffuse, intermediate and focal ( $p=0.60$ ). The comparison of CEHA and CAEH has shown that in CEHA 5 cases were not stained, 5 cases were focally stained, diffuse or intermediate stain were not observed, in CAEH 2 cases were not stained and 4 cases were diffusely stained ( $\mathrm{p}=0.02$ ) (Figure 1C). In ECG II while all the patients sections were stained by CD117, diffuse $(n=4)$ and intermediate $(n=3)$ (Figure 2) in ECG

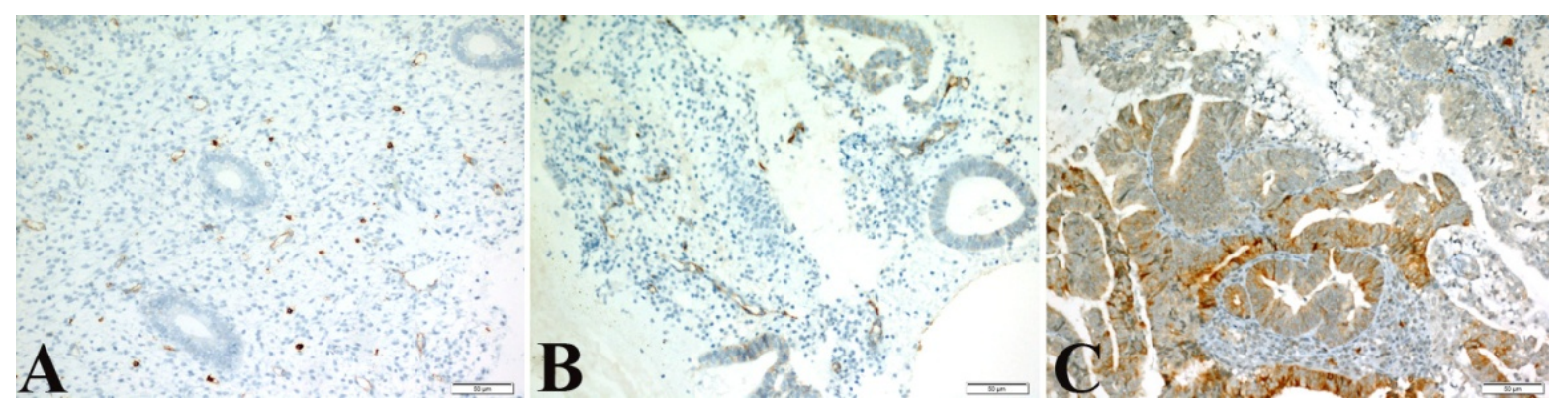

Figure 1. Immunoreactivity of c-kit in endometrial tissues. A) c-Kit immunoreactivity in cytoplasmic mast cells used as positive control group in normal endometrium (x200) B) Weak cytoplasmic c-kit immunostaining in the cells of a simple endometrial hyperplasia without atypia (x200) C) Strong cytoplasmic staining in complex endometrial hyperplasia with atypia (x200) 

Table 1. CD 117 Immunostaining in Endometrial Tissues

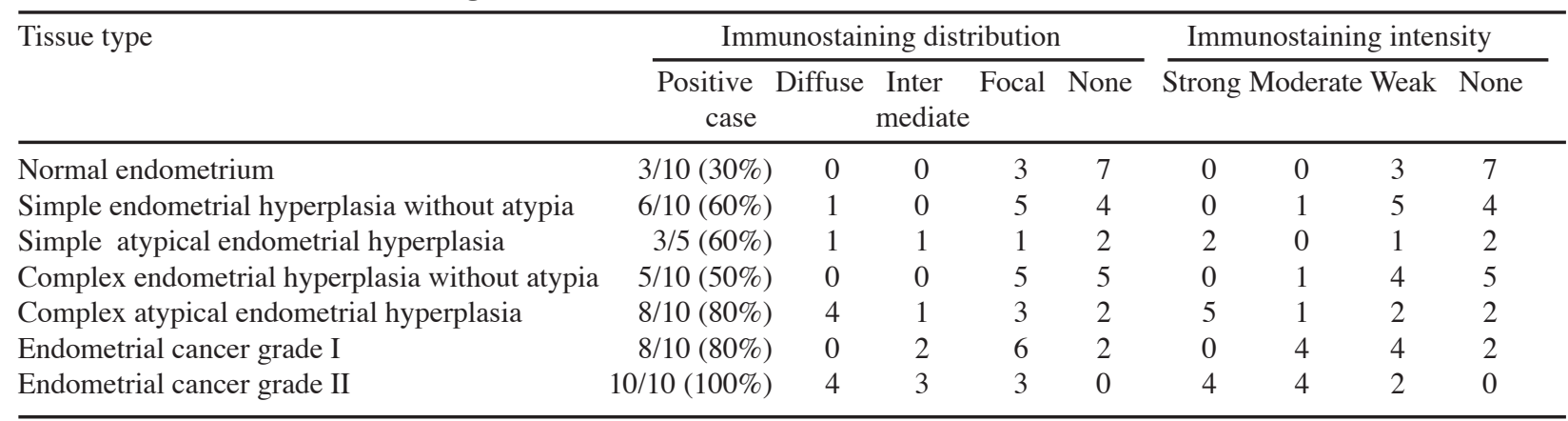

\section{Table 2. Hematologic Parameters in All Groups}

\begin{tabular}{|c|c|c|c|c|}
\hline Tissue type & MPV & Plt & $\mathrm{Hb}$ & Wbc \\
\hline Normal endometrium & $7.1 \pm 1.2$ & $253.3 \pm 80.5$ & $12.1 \pm 1.1$ & $7.3 \pm 1.6$ \\
\hline Simple endometrial hyperplasia without atypia & $7.0 \pm 0.8$ & $285.1 \pm 89.6$ & $12.0 \pm 1.6$ & $7.0 \pm 1.7$ \\
\hline Simple atypical endometrial hyperplasia & $7.7 \pm 1.0$ & $263.4 \pm 51.5$ & $11.4 \pm 0.5$ & $7.4 \pm 1.4$ \\
\hline Complex endometrial hyperplasia without atypia & $6.8 \pm 0.6$ & $248.3 \pm 68.2$ & $11.4 \pm 2.2$ & $6.0 \pm 1.9$ \\
\hline Complex atypical endometrial hyperplasia & $8.4 \pm 0.7 * g ¥$ & $289.1 \pm 68.4$ & $11.6 \pm 1.1$ & $7.7 \pm 2.7$ \\
\hline Endometrial cancer grade I & $8.6 \pm 0.8^{\chi^{\top} ¥}$ & $254.5 \pm 60.7$ & $11.4 \pm 1.7$ & $7.2 \pm 1.3$ \\
\hline Endometrial cancer grade II & $8.8 \pm 1.0 x \pi J ¥$ & $264.2 \pm 40.5$ & $12.6 \pm 1.3$ & $7.1 \pm 1.1$ \\
\hline
\end{tabular}

MPV: mean platelet volume; Plt: platelet; Hb: hemoglobin; Wbc: white blood cell; ${ }^{*} \mathrm{p}=0.002,{ }^{\mathrm{x}} \mathrm{p}<0.001$ vs. control group; ${ }^{\mathrm{p}} \mathrm{p}<0.001 \mathrm{vs}$. simple endometrial hyperplasia without atypia group, ${ }^{\pi} \mathrm{p}=0.045 \mathrm{vs}$. simple atypical endometrial hyperplasia group, ${ }^{\sharp} \mathrm{p}<0.001 \mathrm{vs}$. complex endometrial hyperplasia without atypia group

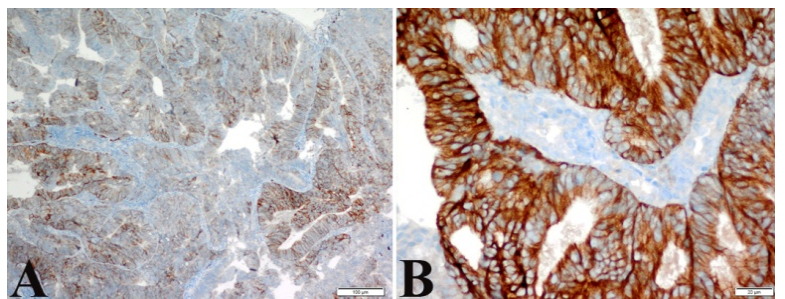

Figure 2. c-Kit Immunoreactivity of Endometrial

Cancer. A) Diffuse cytoplasmic immunostaining in the cells of endometrial cancer (x200) B) Strong cytoplasmic immunostaining in the cells of endometrial cancer $(x 400)$

I, 2 cases were not stained all, there was no diffuse stain and 6 cases were stained focally. There was statistically significant difference between ECG II and ECG I ( $p=0.01$ ). While NE had not statistically a significant relation with SEHA (p:0.15), SAEH $(p=0.14)$ and CEHA $(p=0.37)$, CAEH $(p=0.009)$, ECG I $(p=0.01)$ and ECG II $(p<0.001)$ were observe $d$ to be obviously stained by CD117 more than NE. ECG II $(\mathrm{p}=0.006)$ had been stained by CD117 stronger than SEHA. However, there was no significant relation among ECG I $(p=0.35)$, CEHA $(p=0.52)$ and CAEH $(p=0.1)$. SAEH had similar CD117 staining distribution with ECG I ( $\mathrm{p}=0.89)$, ECG II ( $\mathrm{p}=0.16)$, CEHA $(\mathrm{p}=0.31)$ and $\mathrm{CAEH}(\mathrm{p}=0.44)$ groups. While there was no statistically significant relation between CEHA and ECG I ( $\mathrm{p}=0.08)$ groups, there was an obvious CD117 staining in ECG II $(p<0.001)$. CAEH showed a similar staining pattern with both ECG I ( $p=0.2)$ and ECG II ( $p=0.47)$. All the characteristics of CD117 immunostaining (distribution and intensity) are shown in Table 1

\section{Immunostaining intensity}

While NE group had very low CD117 immunostaining intensity $(30 \%)$, patients with positive CD117 had obviously weak intensity $(100 \%)$. When compared to NE group SEHA ( $\mathrm{p}=0.15), \mathrm{SAEH}(\mathrm{p}=0.14)$ and CEHA $(\mathrm{p}=0.31)$ of the patients with positivity were observed to have similar intensity. However, CAEH $(\mathrm{p}=0.006), \mathrm{ECG}$ I $(\mathrm{p}=0.01)$ and ECG II $(\mathrm{p}<0.001)$ groups were obviously different regarding the number of positive cases and intensity. While SEHA was found to be similar with both CEHA $(p=0.7)$ and ECG I $(p=0.14)$, it had a stronger intensity than CAEH $(p=0.03)$ and ECG II $(p<0.001)$. When the intensity of SAEH group is compared with the other groups SEHA ( $p=0.47)$, CEHA $(p=0.35)$, CAEH $(p=0.513)$, ECG I $(p=0.899)$ and ECG II $(p=0.305)$ there was no obvious difference.

While CEHA group had similar intensity with ECG I ( $p=0.091)$ group, it had a significant difference with CAEH $(\mathrm{p}=0.02)$ and ECG I $(\mathrm{p}<0.001)$. There was no statistically difference between CAEH and both ECG I $(\mathrm{p}=0.16)$ and ECG II $(\mathrm{p}=0.74)$ groups. There was 2 cases with negative CD117 and the strong intensity had never been seen in positive cases. All the cases of ECG II were CD117 positive and $40 \%$ of them had strong intensity. There was a significant difference between ECG I and ECG II ( $\mathrm{p}=0.01)$. All the results are shown in Table 1.

\section{MPV and other hematologic parameters}

When compared with NE MPV levels of CAEH $(\mathrm{p}=0.002)$, ECG I $(\mathrm{p}<0.001)$ and ECG II $(\mathrm{p}<0.001)$ were significantly high. MPV levels of both SEHA and CEHA were obviously higher than CAEH $(\mathrm{p}<0.001)$, ECG I $(\mathrm{p}<0.001)$ and ECG II $(\mathrm{p}<0.001)$ groups. All the results are shown in Table 2.

\section{Correlation analysis}

A positive correlation was found between the wide extension and MPV $(\mathrm{r}=0.296, \mathrm{p}=0.01)$. A positive correlation was found also between intensity and MPV ( $r=0.328, p=0.008)$. Platelet, hemoglobin and WBC levels had no correlation with the intensity of immunohistochemical staining. 


\section{Discussion}

We have found that CAEH, ECG I and ECG II groups to have more cases with positive stain and more intensity in comparison with normal endometrial tissue. Additionally all cases of ECG II had CD117 positive staining and most cases had diffuse and intermediate distribution, strong and moderate intensity in regard to CD117 positivity. Diffuse distribution and strong intensity were observed obviously in CAEH and ECG II groups. According to these findings CD117 positivity may be useful in the diagnosis and follow up EC and CAEH. Additionally CD117 staining may be useful in the differentiation of $\mathrm{EC}$ and $\mathrm{CAEH}$ from normal endometrial tissues. Interestingly, we have found that MPV levels of SAEH and CAEH groups to be higher than NE, SEHA and CEHA groups. Both ECG I and ECG II groups had higher MPV levels than the other groups. However, there was no statistically significance between ECG I and ECG II groups. This may be related to limited number of cases. This study has shown that MPV level may be helpful in differentiation between hyperplasia with atypia and hyperplasia without atypia.

In literature it has been reported that c-kit expression may be seen in various tissue cells including thyroid, parotid, dermal sweat glands, esophageal glands, breast epithelium, renal tubules, testis, ovary, surface epithelial and hematologic stem cells (Natali et al., 1992; Natali et al., 1995; Andreadis et al., 2006; Terada 2012). Various studies have investigated whether c-kit is stained in normal endometrium and EC tissues. Interestingly, these studies have reported different results. While a previous study has reported that c-kit is not expressed in normal endometrial tissues (Lammie et al., 1994). Another study has reported that c-kit is expressed highly (60\%) in NE and SEHA and additionally they reported that diffuse and strong immunostaining to be found in SEHA and CEHA cases (Yilmaz et al., 2012). In another study has reported c-kit to be positive in normal endometrial tissues in a rate of 79-93\% and a diffuse intensity and strong immunostaining to be present in these cases (Elmore et al., 2001). In our study we have found c-kit to be lower than previous studies and to be only positive in $30 \%$ of cases with normal endometrial tissues and to be especially weakly stained by immunostaining (Elmore et al., 2001; Yilmaz et al., 2012). Additionally, in the current study cases of SEHA and CEHA were stained weakly by immunostaining.

c-Kit expression is observed in many solid cancers such as pancreas, lung, breast and ovary (Trinh et al., 2012; Salomonsson et al., 2013; Afsar et al., 2014; Jansson et al., 2014). It is known that c-kit expression has mutagenic effect. It may lead to tumor development by impairing cellular growth regulation (Miettinen et al., 2005) . Interestingly, a previous study has reported that EC cases to have c-kit expression in a very low rate as 16\% (Yilmaz et al., 2012). However, other studies have reported higher c-kit expression in EC cases. Elmore et al. (2001) have found c-kit expression in EC cases to be $44 \%$ (Elmore et al., 2001) In a study has shown 57 cases of endometrioid type EC to have $60 \% \mathrm{c}$-kit expression and 10 cases of serous type EC to have $80 \%$ c-kit expression (Scobie et al., 2003). It has been reported that in both types especially focal and intermediate immunostaining were mostly observed. Similarly, another study has reported c-kit expression to be 50\% ECG I and ECG II (Menczer et al., 2005). In our study, also we have found c-kit expression to be intensively in CAEH, ECG I and ECG II especially it was observed to have diffused staining in CAH and ECG II. The diffuse pattern of c-kit expression may indicate more aggressive course of the tumor. Scobie et al. especially after detecting weak immunostaining of c-kit expression, they emphasized that the results of the studies conducted on low number of subjects to be exaggerated (Scobie et al., 2003). On the other hand, the other studies were cross-sectional and the have even reported c-kit expression in normal endometrial tissues. However, there were no long-term follow-up studies. En important point is that patients with intensive c-kit expression who have other risk factors are at greater risk of EC. We speculate that small population studies might be predictive for the diagnosis of new EC cases. Thus the investigation of c-kit expression may be necessary as a routine screening test in the future.

MPV is a useful, simple and inexpensive marker shows young and large thrombocytes in periphery, thus its high level is accused for thrombotic diseases of veins and arteries (Cure et al., 2014). Additionally, MPV was found to be high in the majority of cancer cases and was reported to be a prognostic factor for EC. In addition, MPV was found to be useful in reflecting EC stages (Oge et al., 2013; Varol et al., 2014). In the majority of cancer patients' excessive production of proinflammatory cytokines stimulate the release of young and large thrombocytes from bone marrow into periphery (Matowicka-Karna et al., 2013). In our study, patients with simple/complex hyperplasia with atypia and those with EC were found to have higher MPV level than the other groups. Even though MPV level of ECG II was higher than ECG I it was not statistically significant, this may be as a result of low number of the subjects. Elevated MPV level reflects the number of young and large active thrombocytes, thus production and storage of PDGF may be intensive in these thrombocytes. On the other hand, the PDGFs bind to the protein tyrosine kinase receptors PDGF receptor- $\alpha$ and $-\beta$. PDGF and are responsible for cellular growth and angiogenesis. However, excess production of PDGF is thought to be responsible carcinogenesis (Wang et al., 2014; Zhu et al., 2014). Besides being accused for many cancers Ding et al. have found PDGF expression to be high in EC patients and they reported the expression of PDGF to be poor prognostic factor (Ding et al., 2014). Similarly, c-kit encodes a receptor of tyrosine kinase family (CD117) and has a strong relation with PDGF receptor family (Zhu et al., 2014). Thus, c-kit expression and elevated MPV level may indicate high stimulation of tyrosine kinase and malignancy risk and may be helpful in the diagnosis of tumors. We have found MPV to have a strong relation with c-kit expression and immunostaining. The c-kit positivity and its strong staining rate increase steadily with increasing MPV. In fact, this is not an incidental finding. According to our hypothesis, when young, large and active thrombocytes is increasing in periphery blood cell, these thrombocytes are releasing excessive PDGF; therefore, 
Relationships Between C-Kit Expression and Mean Platelet Volume in Benign, Preneoplastic and Neoplastic Endometrium

it may be elevated by increasing the activation of c-kit expression owing to its member of tyrosine kinase family.

c-Kit expression is observed in low rate in normal endometrial tissue and in cases with hyperplasia without atypia. As c-kit expression is associated with mutagenicity a long term follow up is needed in these patients. c-Kit expression and immunostaining intensity are observed to be intensive in endometrial tissue with atypia and EC. MPV indicates the number of young, large and active thrombocytes. Increased active thrombocytes in periphery leads to excessive production of PDGF thus accelerating both carcinogenesis and increasing c-kit expression a member of tyrosine kinase family. c-Kit expression and MPV level may be reliable screening tests to determine cancer risk in patients with risk factors.

\section{References}

Afsar CU, Gunaldi M, Kum P, et al (2014). Pancreatic carcinoma, thrombosis and mean platelet volume: single center experience from the southeast region of Turkey. Asian Pac J Cancer Prev, 15, 9143-6.

Andreadis D, Epivatianos A, Poulopoulos A et al (2006). Detection of C-KIT (CD117) molecule in benign and malignant salivary gland tumours. Oral Oncol, 42, 57-65.

Cumhur Cure M, Cure E, Yuce S, Yazici T, Karakoyun I, Efe $\mathrm{H}$ (2014). Mean platelet volume and vitamin D level. Ann Lab Med, 34, 98-103.

d'Auriol L, Mattei MG, Andre C, Galibert F (1988). Localization of the human c-kit protooncogene on the q11-q12 region of chromosome 4. Hum Genet, 78, 374-6.

Ding J, Li XM, Liu SL, Zhang Y, Li T (2014). Overexpression of platelet-derived growth factor-D as a poor prognosticator in endometrial cancer. Asian Pac J Cancer Prev, 15, 3741-5.

Dossus L, Lukanova A, Rinaldi S et al (2013). Hormonal, metabolic, and inflammatory profiles and endometrial cancer risk within the EPIC cohort--a factor analysis. Am J Epidemiol, 177, 787-99.

Elmore LW, Domson K, Moore JR, Kornstein M, Burks RT (2001). Expression of c-kit (CD117) in benign and malignant human endometrial epithelium. Arch Pathol Lab Med, 125, 146-51.

Jansson S, Bendahl PO, Grabau DA et al (2014). The three receptor tyrosine kinases c-KIT, VEGFR2 and PDGFR $\alpha$, closely spaced at 4q12, show increased protein expression in triple-negative breast cancer. PLoS One, 9, 102176.

Inoue M, Kyo S, Fujita M, Enomoto T, Kondoh G (1994). Coexpression of the c-kit receptor and the stem cell factor in gynecological tumors. Cancer Res, 54, 3049-53.

Lammie A, Drobnjak M, Gerald W, Saad A, Cote R, CordonCardo C (1994). Expression of c-kit and kit ligand proteins in normal human tissues. J Histochem Cytochem, 42, 1417-25.

Linkov F, Edwards R, Balk J et al (2008). Endometrial hyperplasia, endometrial cancer and prevention: gaps in existing research of modifiable risk factors. Eur J Cancer, 44, 1632-44.

Matowicka-Karna J, Kamocki Z, Polinska B, Osada J, Kemona H (2013). Platelets and inflammatory markers in patients with gastric cancer. Clin Dev Immunol, 2013, 401623.

Menczer J, Kravtsov V, Levy T, et al (2005). Expression of c-kit in uterine carcinosarcoma. Gynecol Oncol, 96, 210-5.

Miettinen M, Lasota J (2005). KIT (CD117): a review on expression in normal and neoplastic tissues, and mutations and their clinicopathologic correlation. Appl Immunohistochem Mol Morphol, 13, 205-20.
Natali PG, Berlingieri MT, Nicotra MR et al (1995). Transformation of thyroid epithelium is associated with loss of c-kit receptor. Cancer Res, 55, 1787-91.

Natali PG, Nicotra MR, Sures I, Santoro E, Bigotti A, Ullrich A (1992). Expression of c-kit receptor in normal and transformed human nonlymphoid tissues. Cancer Res, 52, 6139-43.

Oge T, Yalcin OT, Ozalp SS, Isikci T (2013). Platelet volume as a parameter for platelet activation in patients with endometrial cancer. J Obstet Gynaecol, 33, 301-4.

Park Y, Schoene N, Harris W (2002). Mean platelet volume as an indicator of platelet activation: methodological issues. Platelets, 13, 301-6.

Salomonsson A, Jonsson M, Isaksson S et al (2013). Histological specificity of alterations and expression of KIT and KITLG in non-small cell lung carcinoma. Genes Chromosomes Cancer, 52, 1088-96.

Scobie JV,Acs G, Bandera CAet al (2003). C-kit immunoreactivity in endometrial adenocarcinomas and its clinicopathologic significance. Int J Gynecol Pathol, 22, 149-55.

Terada T (2012). Protein expression and gene mutation status of KIT and PDGFRA in renal cell carcinoma. Histol Histopathol, 27, 297-302.

Trinh DT, Shibata K, Hirosawa T et al (2012). Diagnostic utility of CD117, CD133, SALL4, OCT4, TCL1 and glypican-3 in malignant germ cell tumors of the ovary. J Obstet Gynaecol Res, 38, 841-8.

Varol E, Ozaydin M (2014). Confounding factors should be considered in mean platelet volume evaluation in endometrial cancer. J Obstet Gynaecol, 34, 455.

Wang Y, Qiu H, Hu W, Li S, Yu J (2014). Over-expression of platelet-derived growth factor-D promotes tumor growth and invasion in endometrial cancer. Int J Mol Sci, 15, 4780-94.

Weigel MT, Ghazoui Z, Dunbier A, et al (2012). Preclinical and clinical studies of estrogen deprivation support the PDGF/ Abl pathway as a novel therapeutic target for overcoming endocrine resistance in breast cancer. Breast Cancer Res, $14,78$.

Xiao H, Wang J, Liu Y, Li L (2014). Relative influence of c-Kit expression and epidermal growth factor receptor gene amplification on survival in patients with non-small cell lung cancer. Oncol Lett, 8, 582-8.

Yarden Y, Kuang WJ, Yang-Feng T et al (1987). Human protooncogene c-kit: a new cell surface receptor tyrosine kinase for an unidentified ligand. $E M B O J, \mathbf{6}, 3341-51$.

Yilmaz E, Celik O, Simsek Y et al (2012). c-Kit proto-oncogene expression in endometrial hyperplasia and endometrial cancer. Arch Gynecol Obstet, 286, 197-200.

Zhu Y, Wang Y, Guan B et al (2014). C-kit and PDGFRA gene mutations in triple negative breast cancer. Int J Clin Exp Pathol, 7, 4280-5. 